\title{
On-surface Synthesis of [7]triangulene Quantum Ring via Antidot Engineering
}

Jie Su ${ }^{\dagger[\mathrm{a}]}$, Wei Fan ${ }^{\dagger[\mathrm{a}]}$, Pingo Mutombo ${ }^{\dagger[\mathrm{c}]}$, Xinnan Peng ${ }^{[\mathrm{a}]}$, Shaotang Song ${ }^{[\mathrm{a}]}$, Mykola Telychko ${ }^{[a]}$, Pavel Jelínek $^{*[c, d]}$, Jishan $\mathrm{Wu}^{*[a]}$ and Jiong $\mathrm{Lu}^{*}[\mathrm{a}, \mathrm{b}]$

${ }^{[a]}$ Department of Chemistry, National University of Singapore, 3 Science Drive 3, Singapore 117543, Singapore.

${ }^{[b]}$ Centre for Advanced 2D Materials (CA2DM), National University of Singapore, 6 Science Drive 2, Singapore 117546, Singapore.

${ }^{[c]}$ Institute of Physics, The Czech Academy of Sciences, Prague 16200, Czech Republic.

${ }^{[\mathrm{d}]}$ Regional Centre of Advanced Technologies and Materials, Palacký University, Olomouc 78371, Czech Republic.

${ }^{\dagger}$ These authors contributed equally to this work

*Corresponding authors. Email: jelinekp@fzu.cz (P. Jelínek); chmwuj@nus.edu.sg (J. Wu); chmluj@nus.edu.sg (J. Lu)

\begin{abstract}
The ability to engineer geometrically well-defined antidots in large triangulene homologues allows for creating an entire family of triangulene quantum ring (TQR) structures with tunable high-spin ground state and magnetic ordering, crucial for next-generation molecular spintronic devices. Herein, we report the synthesis of an open-shell [7]triangulene quantum ring ([7]TQR) molecule on $\mathrm{Au}(111)$ through the surface-assisted cyclodehydrogenation of a rationally-designed kekulene derivative. Bond-resolved scanning tunneling microscopy (BR-STM) unambiguously imaged the molecular backbone of a single [7]TQR with a triangular zigzag edge topology, which can be viewed as [7]triangulene decorated with a coronene-like antidot in the molecular centre. Additionally, $\mathrm{d} I / \mathrm{d} V$ mapping reveals that both inner and outer zigzag edges contribute to the edge-localized and spin-polarized electronic states of [7]TQR. Both experimental results and spin-polarized density functional theory calculations indicate that [7]TQR
\end{abstract}


retains its open-shell septuple ground-state $(S=3)$ on $\mathrm{Au}(111)$. This work demonstrates a new route for the design of high-spin graphene quantum rings as the key components for future quantum devices.

\section{Introduction}

Open-shell graphene nanostructures (GNs) have attracted tremendous interest due to their unique electronic and magnetic properties, which make them promising candidates for carbon-based molecular spintronics. ${ }^{[1-4]}$ The magnetic ordering of these open-shell GNs can be precisely tailored via engineering their topological structures and edge configurations. ${ }^{[5,6]}$ In particular, cutting triangular motifs along the zigzag orientation of graphene creates an entire family of triangulenes with high-spin ground states and large spin quantum number scaling linearly with the number of carbon atoms at their zigzag edges. ${ }^{[7-9]}$ The ground-state spin quantum number of the triangulenes is associated with the sublattice imbalance, as predicted by Ovchinnikov's rule and Lieb's theorem for bipartite lattices. ${ }^{[10,11]}$ As such, the ground-state spin quantum number $(S)$ of triangulenes is equal to $\frac{\left|N_{A}-N_{B}\right|}{2}$, where $N_{A}$ and $N_{B}$ represent the number of carbon atoms from the two interpenetrating sublattices (A and B), respectively.

Recently, [3]-, [4]- and [5]triangulene molecules have been successfully fabricated on different surfaces and their chemical structures and spin-polarized edge states have been characterized by scanning probe techniques. ${ }^{[12-14]} \mathrm{A}$ further extension of triangulene molecules to a larger system not only increases their ground-state spin quantum number but also offers higher feasibility for the device fabrication. ${ }^{[15]}$ Apart from tuning the size of triangulenes, topological engineering via creating an antidot (denoted as antidot engineering) in large triangulene homologues provides an alternative approach to fabricate a series of intriguing triangulene quantum rings (TQRs) with tailored spin quantum number and magnetic ordering associated with both inner and outer zigzag edges. ${ }^{[16-19]}$ Compared to the triangulene counterparts, the presence of an antidot in TQRs results in a larger number of zigzag edges per unit area. Moreover, the spin-polarized energy gap and spin density distribution of TQRs, together with the magnetic ordering in both inner and outer zigzag edges, can be precisely controlled via tailoring the topology of antidots. ${ }^{[20-22]}$ Therefore, antidot engineering constitutes a flexible and versatile strategy 
towards the fabrication of a plethora of novel magnetic quantum nanostructures, crucial to unlocking their full potential in next-generation spintronics applications.

Analogously to triangulenes, the ground-state spin quantum number of TQRs can also be predicted by Ovchinnikov's rule by evaluating their sublattice imbalance. As illustrated in Figure 1a, excavating a coronene-moiety from the centre of an intact [7]triangulene molecule (Figure 1a, right) generates a [7]TQR molecule containing a hexagonal coronene-like antidot (Figure 1a, middle right). Notably, the as-removed hexagonal motif consists of the equal number of two sublattices (A- and B-type). As a result, the ground-state spin quantum number $(S=3)$ is preserved after introducing such a hexagonal antidot in [7]triangulene. In contrast to [7]triangulene, the presence of inner zigzag edges in [7]TQR is expected to modulate its spin density and magnetic ordering in this system. ${ }^{[21]}$ Similarly, larger TQRs also retain the same high spin quantum after removing hexagonal antidots (e.g., high-order coronene-like moieties) in the triangulene hosts (e.g., [10]TQR, Figure 1b, right). By contrast, incorporating triangular antidots into triangulenes can reduce the sublattice imbalance and thus lower their ground-state spin quantum numbers, as exemplified in the case of [8]TQR and [9]TQR (Figure 1b, left). Both of them have the same groundstate spin quantum number of $S=3$, lower than that of [8]triangulene $\left(S=\frac{7}{2}\right)$ and [9] triangulene $(S=4)$, respectively. In this case, the ground-state spin quantum number $S$ only depends on the width of the ring, as defined by the number of benzene units $\left(N_{W}\right)$ between outer and inner edges: $S=3\left(N_{W}+1\right)$. ${ }^{[21]}$ Therefore, the ground-state spin quantum number of TQRs can be modulated by tailoring the topology of the antidots, including the lateral size (Figure 1b, right) and width of the ring (Figure 1b, left).

\section{Results and Discussions}

To demonstrate the concept of antidot engineering, we designed a new precursor $\mathbf{1}$ containing a kekulene-core attached by three 2,6-dimethylphenyl substituents at meso- positions of three alternating edges. The kekulene is well-known for its annelated macrocyclic topology that provides an ideal platform for antidot engineering. ${ }^{[23-27]}$ The dehydrogenation of methyl groups of precursor 1 generates benzylic 
radicals, which then couple with the neighbouring carbon atoms of kekulene-core. Subsequently, an aromaticity-driven ring-closure reaction leads to the formation of [7]TQR (Figure 1a). Precursor 1 was obtained via multiple solution-phase synthetic steps as inspired by our previous work (see details in the Supporting Information). ${ }^{[28]}$ In brief, a macrocyclic oligo( $m$-phenylene) intermediates 7 carrying six vinyl ether groups was first synthesized by Suzuki coupling reaction. The subsequent $\mathrm{Bi}(\mathrm{OTf})_{3}$ catalyzed cyclization reaction of 7 in 1,2-dichloroethane at $90{ }^{\circ} \mathrm{C}$ led to the formation of the 2,6-dimethylphenylsubstituted kekulene 1 in $76 \%$ yield. The structure of the precursor 1 was confirmed by ${ }^{1} \mathrm{H} \mathrm{NMR},{ }^{13} \mathrm{C}$ NMR spectra, and mass spectrum.

Precursor 1 was first deposited onto $\mathrm{Au}(111)$ at a submonolayer coverage, under ultra-high vacuum conditions. It exhibits a three-lobed appearance in the STM image attributed to its three-fold structural symmetry (Figure S1). Subsequently, a post-annealing of $\mathrm{Au}(111)$ substrate at $550 \mathrm{~K}$ for 10 minutes triggers the surface-assisted cyclodehydrogenation of $\mathbf{1}$ towards the formation of [7]TQR. ${ }^{29]}$ An overview STM image captures that as-formed products contain both isolated triangular-shaped molecules and irregular oligomers presumably arising from intermolecular coupling on $\mathrm{Au}(111)$ during thermal annealing process (Figure 2a, Figure S1). Isolated triangular-shaped molecules constitute approximately $26 \%$ of all the products, as estimated by a statistical analysis of multiple STM images. In addition, a magnified STM image of triangular-shaped products with a metallic tip resolves a dim hole-like feature in the molecular centre (Figure $2 \mathrm{~b}$ and Figure $\mathrm{S} 1$ ) and characteristic edge-localized nodal patterns, resembling the patterns observed at the zigzag edges of the zigzag-edged graphene nanostructures in the previous reports. ${ }^{[13,14,29,30]}$ To probe the chemical structure of an isolated product $\mathbf{1}$, we performed bondresolved STM (BR-STM) imaging with a carbon monoxide-functionalized tip (CO-tip). ${ }^{[31-34]}$ The BRSTM imaging was conducted at constant-height mode with reduced tip-sample distances (i.e. Pauli repulsion regime), wherein the $\mathrm{CO}$ molecule undergoes a lateral relaxation over the areas with high electron density (repulsive) areas (chemical bonds), which modulates the overall tunneling conductance between tip and sample, resulting in sharp features associated with the chemical bonds in the current 
image. ${ }^{[35,36]}$ The corresponding BR-STM image of a single triangular-shaped molecule (Figure 2c) unambiguously resolves the molecular backbone consisting of twelve fused benzene rings with a clearcut hexagonal antidot (a diameter of $5.7 \AA$ ) in the molecular centre. Furthermore, the flat adsorption geometry of final products on $\mathrm{Au}(111)$ indicates the absence of any chemical bonding of molecule to the surface. These observations confirm the successful synthesis of the [7]TQR on Au(111).

To unveil the electronic structure of [7]TQR, we acquired differential conductance spectra (dI/dV) over a single molecule on $\mathrm{Au}(111)$ using a calibrated metallic tip. The characteristic point $\mathrm{d} I / \mathrm{d} V$ spectra collected at the corner (red curve) and the edge centre (blue curve) of a [7]TQR along with the reference spectrum recorded on bare $\mathrm{Au}(111)$ (grey dashed curve), are presented in Figure 3a. The $\mathrm{d} I / \mathrm{d} V$ spectrum collected at the edge corner of the molecule (red cross in the inset image in Figure 3a) shows two pronounced peaks at $-0.61 \mathrm{~V}( \pm 0.05 \mathrm{~V})$ and $+0.91 \mathrm{~V}( \pm 0.05 \mathrm{~V})$, presumably corresponding to the positive and negative ion resonances (PIR and NIR), respectively. We also observed a weak "bump" feature around $-0.3 \mathrm{~V}( \pm 0.05 \mathrm{~V})$ that overlaps with the $\mathrm{Au}(111)$ surface states, which can be better resolved in $\mathrm{d} I / \mathrm{d} V$ spectrum acquired in a smaller bias range (Figure S3). In addition, a prominent peak at $-1.47 \mathrm{~V}$ $( \pm 0.05 \mathrm{~V})$ and a substantial rise above $+2.0 \mathrm{~V}$ can be observed at both $\mathrm{d} I / \mathrm{d} V$ spectra acquired at the corner and the edge centre.

In order to gain a more in-depth insight into the electronic structures of [7]TQR, we performed spinpolarized density functional theory (DFT) calculations. ${ }^{[37,38]}$ Firstly, our calculations reveal that the septuple ground-state $(S=3)$ of a freestanding [7]TQR is lower in energy by 378 and $448 \mathrm{meV}$ than its $S=1$ open-shell states, respectively (Figure 2d, Figure S2), consistent with the theoretical predictions of the triangulene systems. ${ }^{[13,14]}$ Figure $3 b$ presents the calculated single-particle DFT spin-polarized energy level diagram of a freestanding [7]TQR in the ferromagnetic septuple state $(S=3)$. The frontier orbitals of a neutral [7]TQR (Figure 3a) contain six singly occupied molecular orbitals (denoted as SOMOs including spin-up $\psi_{3 \uparrow}-\psi_{8 \uparrow}$ ) and six singly unoccupied molecular orbitals (denoted as SUMOs including spin-down $\psi_{3 \downarrow}-\psi_{8 \downarrow}$ ) with the corresponding wave function plots shown in Figure 3d. It is noted that a 
large energy splitting of $200 \mathrm{meV}$ between $\psi_{3 \uparrow}-\psi_{5 \uparrow}$ and $\psi_{6 \uparrow}-\psi_{8 \uparrow}$ of SOMOs leads to a double peak feature associated to SOMOs in density of states (DOS) (Figure 3c). This is consistent with our experimental observations of a pair of occupied electronic states at $-0.61 \mathrm{~V}$ and $-0.3 \mathrm{~V}$, which thus can be assigned as $\mathrm{SOMO}_{1}$ and $\mathrm{SOMO}_{2}$ (Figure 3c), respectively. In contrast to SOMOs, a reduced energy splitting between SUMOs leads to a single peak feature of SUMOs-derived DOS, in agreement with the experimental observation of a single broad peak located at $+0.91 \mathrm{~V}$ (labelled as SUMOs in Figure $3 \mathrm{c}$ ). Such a discrepancy between SOMOs and SUMOs-derived DOS features can be attributed to their different degrees of orbital degeneracy (Figure 3b). According to the energy ordering of these molecular orbitals, a sharp peak at $-1.47 \mathrm{~V}( \pm 0.05 \mathrm{~V})$ and a substantial rise above $+2.0 \mathrm{~V}$ can be assigned to HOMO1 (highest occupied molecular orbital) and LUMO+1 (lowest unoccupied molecular orbital) derived from $\psi_{1 \downarrow}-\psi_{2 \downarrow}$ and $\psi_{9 \uparrow}-\psi_{10 \uparrow}$, respectively.

To resolve the spatial distribution of these molecular orbitals, we performed the differential conductance mapping at the corresponding energetic positions of the aforementioned peaks (Figure 4ae). $\mathrm{d} I / \mathrm{d} V$ maps collected at $-0.61 \mathrm{~V}$ (Figure $4 \mathrm{~b}$ ), $-0.3 \mathrm{~V}$ and $+0.91 \mathrm{~V}$ (Figure $4 \mathrm{~d}$ ) reveal the edgelocalization and the characteristic nodal patterns for both the outer and inner edges of [7]TQR, wherein the nodal patterns of outer edges resemble that of $\pi$-extended triangulenes in previous reports. ${ }^{[13,14]}$ As for the triangulenes, SOMOs and SUMOs-derived states often show the identical spatial patterns. However, it is noted that $\mathrm{d} I / \mathrm{d} V$ maps acquired at $-0.61 \mathrm{~V}\left(\mathrm{SOMO}_{1}\right),-0.30 \mathrm{~V}\left(\mathrm{SOMO}_{2}\right)$, and $+0.91 \mathrm{~V}$ (SUMOs) exhibit slightly different spatial patterns. Such a difference can be attributed to the different frontier orbitals comprising $\mathrm{SOMO}_{1}, \mathrm{SOMO}_{2}$ and $\mathrm{SUMOs}$, respectively. Notably, SOMOs with a lower degree of degeneracy split into two sub-sets of nearly-degenerate orbitals $\left(\psi_{3 \uparrow}-\psi_{5 \uparrow}, \mathrm{SOMO}_{1}\right.$ and $\psi_{6 \uparrow}-$ $\psi_{8 \uparrow}, \mathrm{SOMO}_{2}$ ) (Figure $3 \mathrm{~b}$ and $\mathrm{c}$ ), resulting in slightly different patterns in the $\mathrm{d} I / \mathrm{d} V$ maps. All the key features of experimental $\mathrm{d} I / \mathrm{d} V$ maps taken at $-0.61 \mathrm{~V}$ and $-0.3 \mathrm{~V}$ (Figure $4 \mathrm{c}$ ) are well reproduced in the calculated $\mathrm{d} I / \mathrm{d} V$ maps involving the orbitals of $\psi_{3 \uparrow}-\psi_{5 \uparrow}\left(\mathrm{SOMO}_{1}\right)$ and $\psi_{6 \uparrow}-\psi_{8 \uparrow}\left(\mathrm{SOMO}_{2}\right){ }^{[39]}$ In contrast to SOMOs, SUMOs of [7]TQR with a higher degree of degeneracy (Figure 3b) yield a single 
peak in $\mathrm{d} I / \mathrm{d} V$ spectrum. A direct comparison between experimental and theoretical $\mathrm{d} I / \mathrm{d} V$ map reveals that orbitals $\psi_{5 \downarrow}$ and $\psi_{6 \downarrow}$ with similar wave function patterns (Figure $3 \mathrm{~d}$ ) have a considerable contribution (high weightage) to the $\mathrm{d} I / \mathrm{d} V$ map of SUMOs. Such observations further imply that these probed orbitals, indeed, correspond to the frontier SOMOs and SUMOs of [7]TQR.

In addition, $\mathrm{d} I / \mathrm{d} V$ map recorded at $-1.47 \mathrm{~V}$ (HOMO-1) reveals a triangle lobe at the centre and characteristic nodal pattern (three bright lobes) at the outer edges. In contrast, $\mathrm{d} I / \mathrm{d} V$ map taken at +2.20 $\mathrm{V}(\mathrm{LUMO}+1)$ exhibits a hexagonal protrusion at the centre and faint feature at the outer edges, suggesting a larger contribution from the inner edge states to LUMO+1. Moreover, the calculated $\mathrm{d} I / \mathrm{d} V$ images involving the spin-down $\psi_{1 \downarrow}-\psi_{2 \downarrow}$ and spin-up $\psi_{9 \uparrow}-\psi_{10 \uparrow}$ orbitals show excellent agreement with $\mathrm{d} I / \mathrm{d} V$ maps taken at $-1.47 \mathrm{~V}(\mathrm{HOMO}-1)$ and $+2.20 \mathrm{~V}(\mathrm{LUMO}+1)$, respectively (Figure $4 \mathrm{f}$ and $\mathrm{j})$.

Lastly, the energy gap of Au-supported [7]TQR is determined to be $1.21 \mathrm{eV}$ based on the energy separation between $\mathrm{SOMO}_{2}$ and SUMOs (Figure 3A), lower than the theoretically predicted value (2.25 $\mathrm{eV}$ ) of a free-standing [7]TQR. This can be attributed to the gap reduction arising from the screening effect from metallic $\mathrm{Au}(111)$ surface, consistent with the observation in $\pi$-extended triangulenes and other similar systems. ${ }^{[13,14,40-44]}$ All these observations discussed above manifest that the septuple magnetic ground states $(S=3)$ of [7]TQR is likely to be retained on $\mathrm{Au}(111)$.

\section{Conclusion}

In summary, we have demonstrated a new synthetic route to use the substituted-kekulene as the precursor for the synthesis of unsubstituted [7]TQR with a coronene-like antidot on $\mathrm{Au}(111)$. BR-STM characterization unambiguously reveals the chemical structure of [7]TQR at the sub-molecular level, while $\mathrm{d} I / \mathrm{d} V$ measurements unveil the spin-polarized electronic states contributed from both inner and outer zigzag edges of [7]TQR. Both experimental data and theoretical calculations suggest that [7]TQR retains its open-shell septuple ground-state $(S=3)$ on $\operatorname{Au}(111)$. The successful synthesis of triangulene quantum ring via antidot engineering opens up a new avenue for the fabrication of high-spin quantum nanostructures towards next-generation quantum devices. 
Acknowledgements: J. Lu acknowledges the support from MOE grants (MOE2017-T2-1-056, MOE2019-T2-2-044, R-143-000-A75-11, R-143-000-B58-114). J. Wu acknowledges financial support from NRF Investigatorship (NRF-NRFI05-2019-0005) and MOE Tier 2 grant (MOE2018-T2-2-094).

This was supported by GACR Expro 20-13692X. P.J. and P.M. acknowledge support of the Czech Academy of Sciences through Praemium Academiae award. Computational resources were provided by the CESNET LM2015042 and the CERIT Scientific Cloud LM2015085, provided under the programme "Projects of Large Research, Development, and Innovations Infrastructures".

\section{Conflict of interest}

The authors declare no conflict of interest.

Keywords: Triangulene quantum ring $\bullet$ antidot engineering $\bullet$ on-surface synthesis $\bullet$ open-shell $\bullet$ scanning probe microscopy

\section{Reference}

[1] Y. Morita, S. Suzuki, K. Sato, T. Takui, Nat. Chem. 2011, 3, 197-204.

[2] W. Han, R. K. Kawakami, M. Gmitra, J. Fabian, Nat. Nanotech 2014, 9, 794-807.

[3] Z. Sun, J. Wu, J. Mater. Chem. 2012, 22, 4151-4160.

[4] W. L. Wang, S. Meng, E. Kaxiras, Nano Lett. 2008, 8, 241-245.

[5] O. V. Yazyev, Rep. Prog. Phys. 2010, 73, 056501-17.

[6] S. Fujii, T. Enoki, Acc. Chem. Res. 2013, 46, 2202-2210.

[7] J. Fernández-Rossier, J. J. Palacios, Phys. Rev. Lett. 2007, 99, 177204-4.

[8] A. D. Güçlü, P. Potasz, P. Hawrylak, Phys. Rev. B 2013, 88, 155429-8.

[9] W. L. Wang, O. V. Yazyev, S. Meng, E. Kaxiras, Phys. Rev. Lett. 2009, 102, 195-4.

[10] A. A. Ovchinnikov, Theoret. Chim. Acta 1978, 47, 297-304.

[11] E. Lieb, Phys. Rev. Lett. 1989, 62, 1201-1204.

[12] N. Pavliček, A. Mistry, Z. Majzik, N. Moll, G. Meyer, D. J. Fox, L. Gross, Nat. Nanotech 2017, 12, 308-311. 
[13] S. Mishra, D. Beyer, K. Eimre, J. Liu, R. Berger, O. Gröning, C. A. Pignedoli, K. Müllen, R. Fasel, X. Feng, P. Ruffieux, J. Am. Chem. Soc. 2019, 141, 10621-10625.

[14] J. Su, M. Telychko, P. Hu, G. Macam, P. Mutombo, H. Zhang, Y. Bao, F. Cheng, Z.-Q. Huang, Z. Qiu, S. J. R. Tan, H. Lin, P. Jelínek, F.-C. Chuang, J. Wu, J. Lu, Sci. Adv. 2019, 5, eaav7717-7.

[15] J. Su, M. Telychko, S. Song, J. Lu, Angew. Chem. Int. Ed. 2020, 75, 2667-13.

[16] H.-X. Yang, M. Chshiev, D. W. Boukhvalov, X. Waintal, S. Roche, Phys. Rev. B 2011, 84, $214404-7$.

[17] T. G. Pedersen, C. Flindt, J. Pedersen, N. A. Mortensen, A.-P. Jauho, K. Pedersen, Phys. Rev. Lett. 2008, 100, 136804-4.

[18] R. Pawlak, X. Liu, S. Ninova, P. D'Astolfo, C. Drechsel, S. Sangtarash, R. Häner, S. Decurtins, H. Sadeghi, C. J. Lambert, U. Aschauer, S.-X, Liu, E. Meyer, J. Am. Chem. Soc. 2020, 142, $12568-12573$.

[19] C. Moreno, M. Vilas-Varela, B. Kretz, A. García-Lekue, M. V. Costache, M. Paradinas, M. Panighel, G. Ceballos, S. O. Valenzuela, D. Peña, A. Mugarza, Science 2018, 360, 199-203.

[20] D. A. Bahamon, A. L. C. Pereira, P. A. Schulz, Phys. Rev. B 2009, 79, 125414-7.

[21] P. Potasz, A. D. Güçlü, O. Voznyy, J. A. Folk, P. Hawrylak, Phys. Rev. B 2011, 83, 1744416.

[22] M. Grujić, M. Tadić, F. M. Peeters, Phys. Rev. B 2013, 87, 085434-6.

[23] H. A. Staab, F. Diederich, Chem. Ber. 1983, 116, 3487-3503.

[24] J. C. Buttrick, B. T. King, Chem. Soc. Rev. 2017, 46, 7-20.

[25] I. Pozo, Z. Majzik, N. Pavliček, M. Melle-Franco, E. Guitián, D. Peña, L. Gross, D. Pérez, J. Am. Chem. Soc. 2019, 141, 15488-15493.

[26] M. Di Giovannantonio, X. Yao, K. Eimre, J. I. Urgel, P. Ruffieux, C. A. Pignedoli, K. Müllen, R. Fasel, A. Narita, J. Am. Chem. Soc. 2020, 142, 12046-12050.

[27] Q. Fan, D. Martin-Jimenez, S. Werner, D. Ebeling, T. Koehler, T. Vollgraff, J. Sundermeyer, W. Hieringer, A. Schirmeisen, J. M. Gottfried, J. Am. Chem. Soc. 2020, 142, 894-899.

[28] W. Fan, Y. Han, S. Dong, G. Li, X. Lu, J. Wu, CCS Chem. 2020, 1445-1452.

[29] P. Ruffieux, S. Wang, B. Yang, C. Sánchez-Sánchez, J. Liu, T. Dienel, L. Talirz, P. Shinde, C. A. Pignedoli, D. Passerone, T. Dumslaff, X. Feng, K. Müllen, R. Fasel, Nature 2016, 531, $489-492$.

[30] L. Talirz, H. Söde, J. Cai, P. Ruffieux, S. Blankenburg, R. Jafaar, R. Berger, X. Feng, K. Müllen, D. Passerone, R. Fasel, C. A. Pignedoli, J. Am. Chem. Soc. 2013, 135, 2060-2063. 
[31] G. D. Nguyen, H.-Z. Tsai, A. A. Omrani, T. Marangoni, M. Wu, D. J. Rizzo, G. F. Rodgers, R. R. Cloke, R. A. Durr, Y. Sakai, F. Liou, A. S. Aikawa, J. R. Chelikowsky, S. Louie, F. Fischer, M. F. Crommie, Nat. Nanotech. 2017, 12, 1077-1082.

[32] J. Li, N. Merino-Díez, E. Carbonell-Sanromà, M. Vilas-Varela, D. G. de Oteyza, D. Peña, M. Corso, J. I. Pascual, Sci. Adv. 2018, 4, eaaq0582-7. Pascual, Phys. Rev. Lett. 2020, 124, 177201.

S. Song, N. Guo, X. Li, G. Li, Y. Haketa, M. Telychko, J. Su, P. Lyu, Z. Qiu, H. Fang, X. Peng, J. Li, X. Wu, Y. Li, C. Su, M. J. Koh, J. Wu, H. Maeda, C. Zhang, J. Lu, J. Am. Chem. Soc. 2020, 142, 13550-13557.

P. Hapala, G. Kichin, C. Wagner, F. S. Tautz, R. Temirov, P. Jelínek, Phys. Rev. B 2014, 90, 1989-9.

P. Hapala, R. Temirov, F. S. Tautz, P. Jelínek, Phys. Rev. Lett. 2014, 113, 226101-5.

V. Blum, R. Gehrke, F. Hanke, P. Havu, V. Havu, X. Ren, K. Reuter, M. Scheffler, Computer Physics Communications 2009, 180, 2175-2196.

J. P. Perdew, M. Ernzerhof, K. Burke, J. Chem. Phys. 1996, 105, 9982-9985.

O. Krejčí, P. Hapala, M. Ondráček, P. Jelínek, Phys. Rev. B 2017, 95, 045407-9.

]

P. Ruffieux, J. Cai, N. C. Plumb, L. Patthey, D. Prezzi, A. Ferretti, E. Molinari, X. Feng, K. Müllen, C. A. Pignedoli, R. Fasel, ACS Nano 2012, 6, 6930-6935.

Y. J. Zheng, Y. L. Huang, Y. Chen, W. Zhao, G. Eda, C. D. Spataru, W. Zhang, Y.-H. Chang, L.-J. Li, D. Chi, S. Y. Quek, A. T. S. Wee, ACS Nano 2016, 10, 2476-2484.

J. B. Neaton, M. S. Hybertsen, S. G. Louie, Phys. Rev. Lett. 2006, 97, 98-4.

Z.-F. Liu, D. A. Egger, S. Refaely-Abramson, L. Kronik, J. B. Neaton, J. Chem. Phys. 2017, 146, 092326-14.

[44] K. S. Thygesen, A. Rubio, Phys. Rev. Lett. 2009, 102, 046802-4. 
a

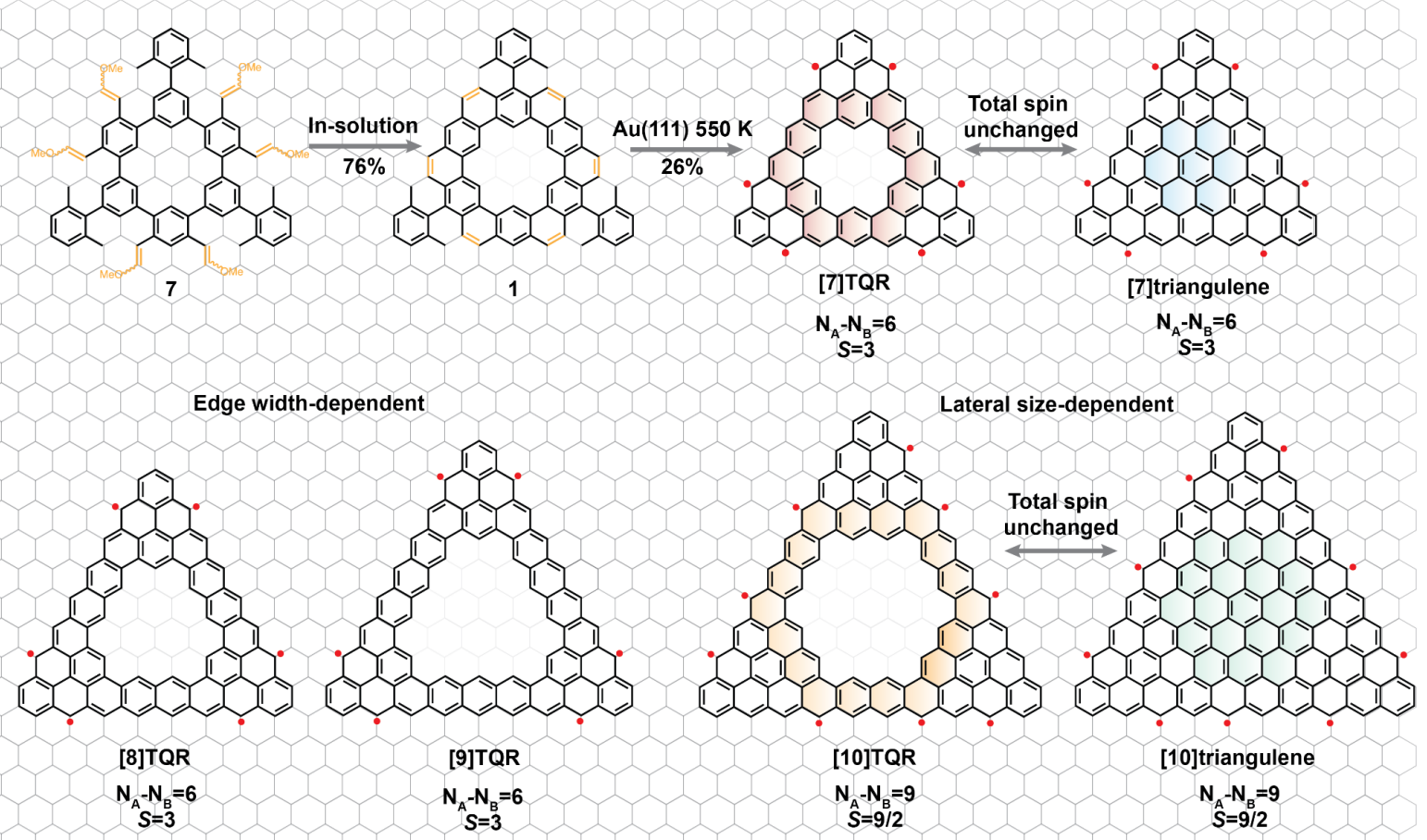

Figure 1. Schematic illustration of the synthesis of [7]TQR via antidot engineering of triangulene molecules. (a) Illustration of the synthetic route towards [7]TQR. [7]TQR with a coronene-like antidot and [7]triangulene have the same ground-state quantum spin number of $S=3$. (b) Engineering of triangular and hexagonal antidots within $\pi$-extended triangulene molecules. Incorporating a triangular antidot in the centre of triangulene modifies the molecules' ground-state spin quantum number. In contrast, introducing a hexagonal antidot in both [7] and [10]- triangulene retains the ground-state spin quantum number 

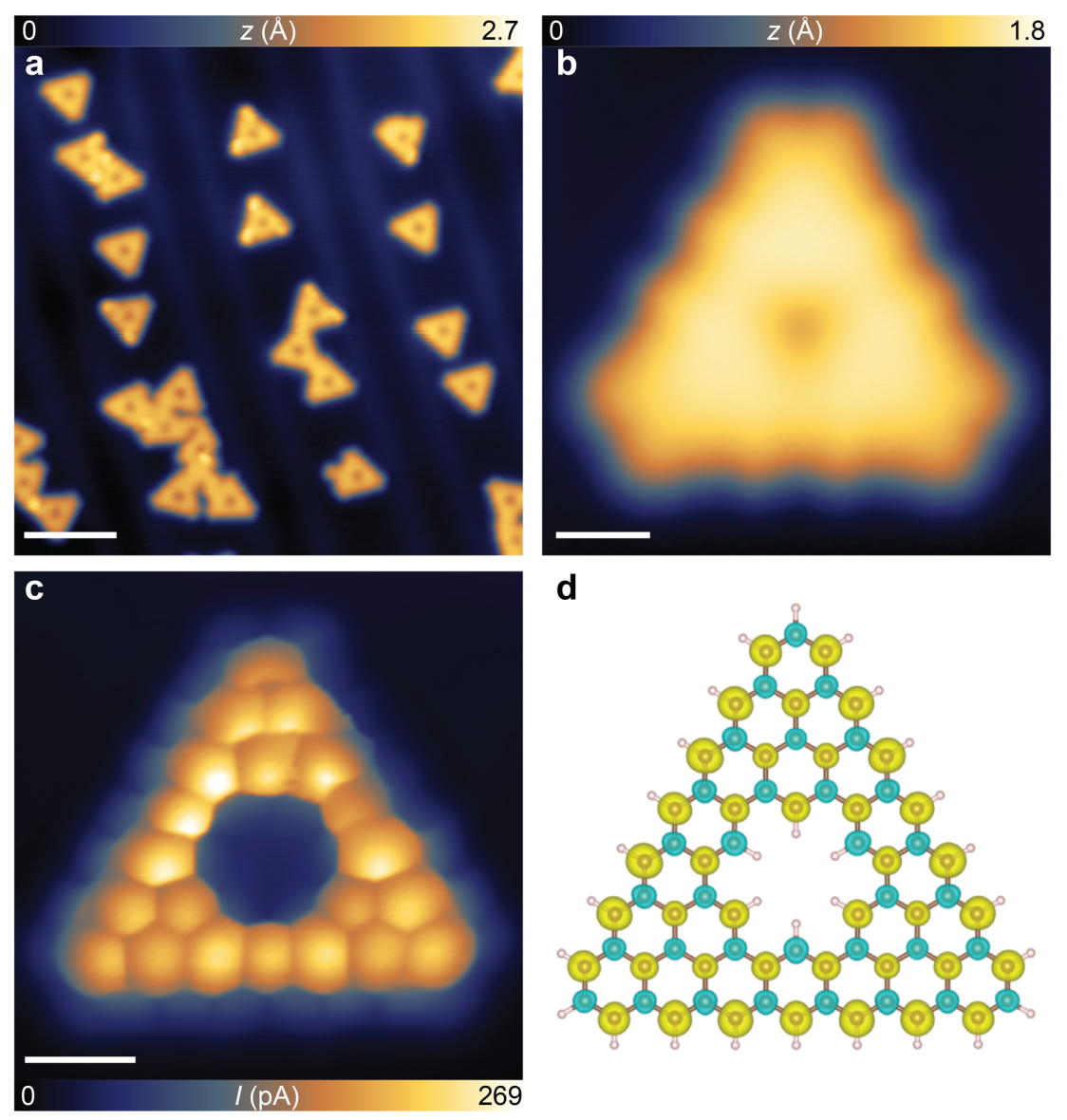

d

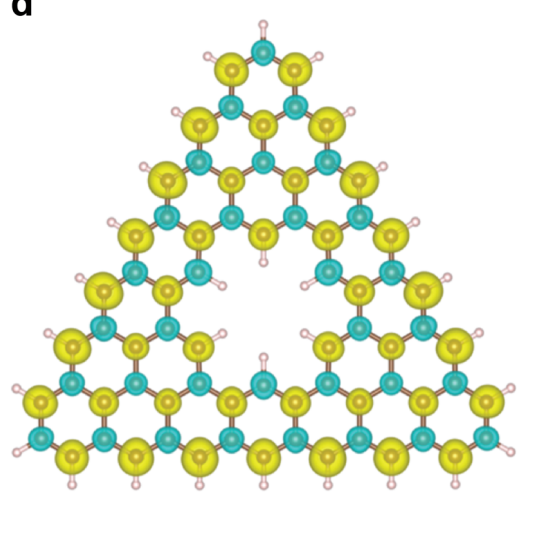

Figure 2. Structural characterization of a single [7]TQR synthesized on $\mathrm{Au}(111)$. (a) Largescale STM image of [7]TQR molecules on $\mathrm{Au}(111)$ after annealing the precursor $\mathbf{1}$ at $550 \mathrm{~K}$ $(V=1.0 \mathrm{~V}, I=300 \mathrm{pA})$. (b) Magnified high-resolution STM image of an individual [7]TQR molecule $(V=-0.61 \mathrm{~V}, I=600 \mathrm{pA})$. (c) Corresponding BR-STM image $(V=5 \mathrm{mV}, \Delta z=-1.1 \AA$, setpoint prior to turn off feedback: $V=50 \mathrm{mV}, I=300 \mathrm{pA}$ ). (d) DFT-calculated spin density distribution of a freestanding [7]TQR molecule. Scale bar: a: $4 \mathrm{~nm}, \mathrm{~b}$ and c: $5 \AA$. 
a

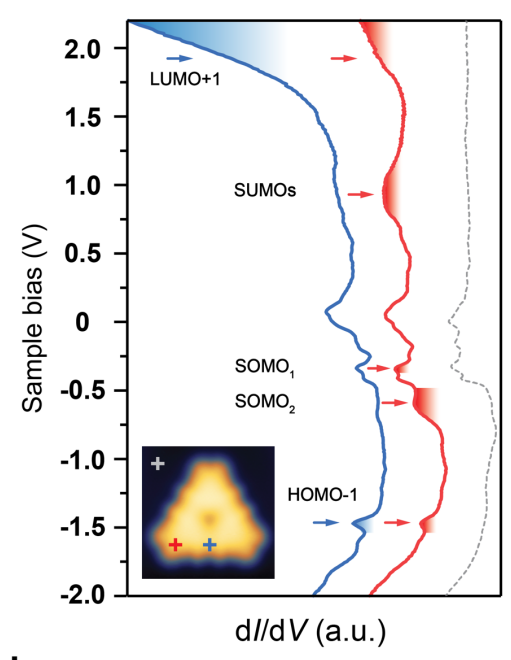

b

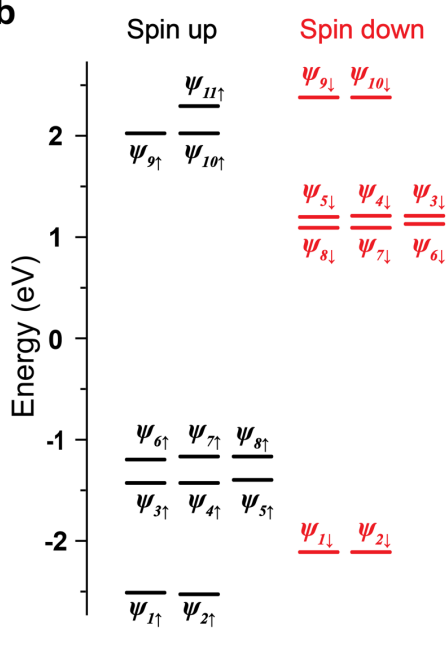

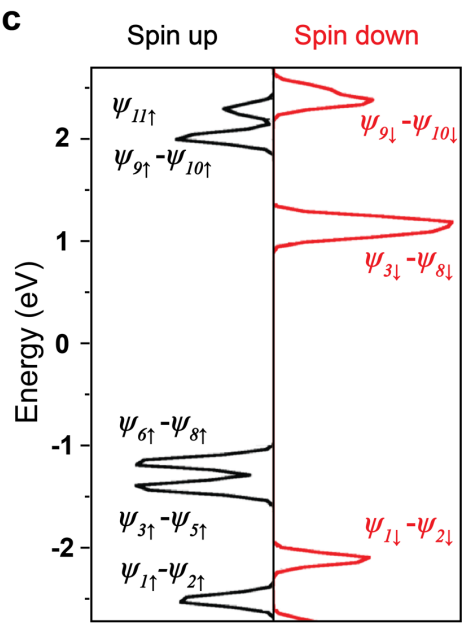

$\operatorname{DOS}($ a.u.)

d
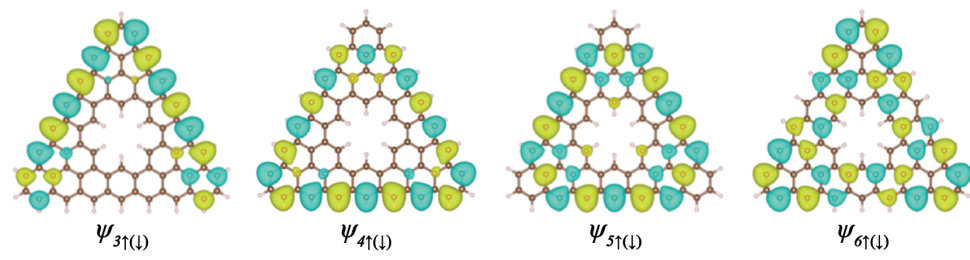

$\psi_{\sigma \uparrow(\downarrow)}$
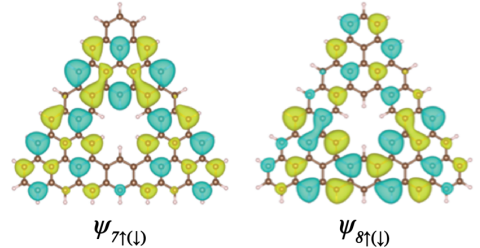

Figure 3. Electronic structure characterization of 7TQR. (a) Point $\mathrm{d} I / \mathrm{d} V$ spectra acquired over different sites of [7]TQR and $\mathrm{Au}(111)$ substrate. $\mathrm{d} I / \mathrm{d} V$ curves taken at the corner (red curve), centre of the edge (blue curve) and taken on $\mathrm{Au}(111)$ (grey-dashed curve). (b) DFT-calculated spin-polarized molecular orbital energy diagram of a freestanding [7]TQR. The black and red symbols refer to spin-up and spin-down states, respectively. (c) Calculated density of states (DOS) of a freestanding [7]TQR. (d) DFT calculated wave functions of six pairs of SOMOs and SUMOs $\left(\psi_{3 \uparrow \downarrow}-\psi_{8 \uparrow \downarrow}\right)$. The blue and green isosurface colours indicate opposite phase of the wave function, respectively. (a.u., arbitrary units). 

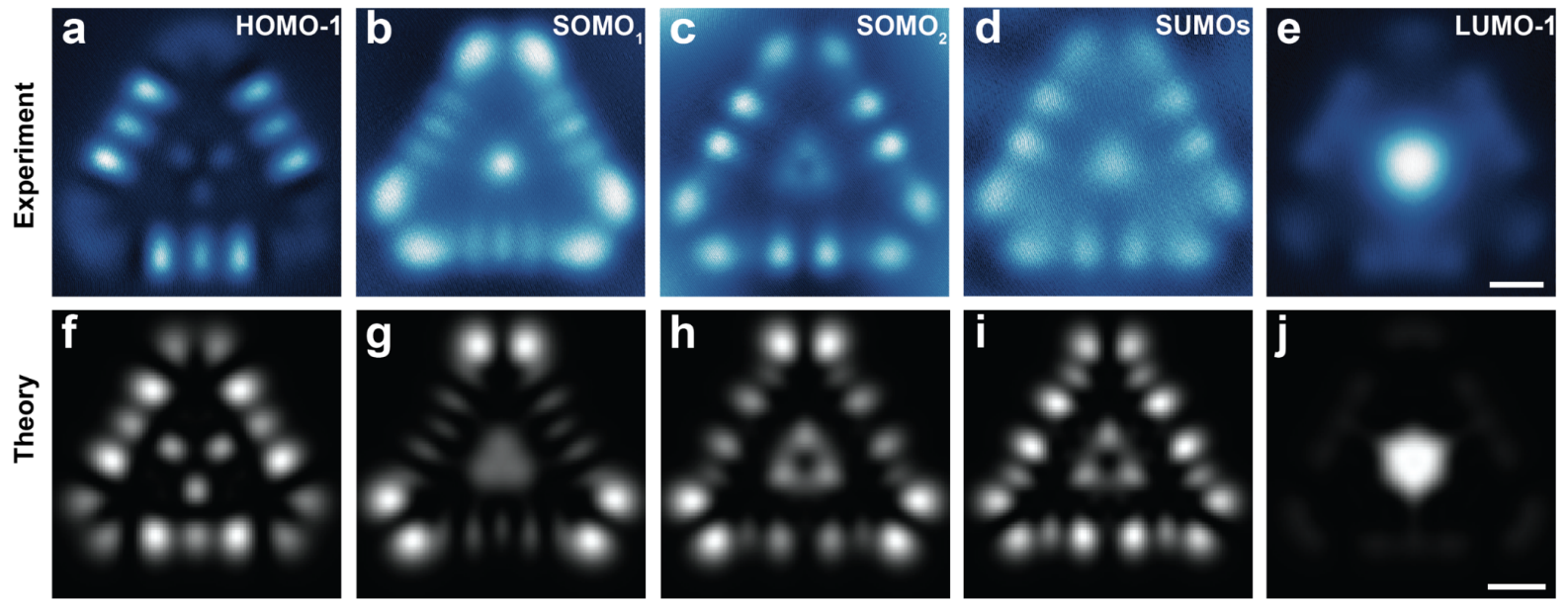

Figure 4. Experimental and calculated $\mathrm{d} I / \mathrm{d} V$ maps of 7TQR. (a)- (e) Constant-current $\mathrm{d} I / \mathrm{d} V$ maps recorded at different energy positions. (a) and (e) refer to the HOMO-1 (-1.47 V) and LUMO+1 (+2.20 V), of [7]TQR respectively. (b-d) and refer to the $\mathrm{SOMO}_{1}(-0.61 \mathrm{~V}), \mathrm{SOMO}_{2}$ $(-0.3 \mathrm{~V})$ and SUMOs (+0.91 V) of 7TQR, respectively. (f)-(j) Simulated $\mathrm{d} I / \mathrm{d} V$ maps acquired at different energy positions corresponding to different sets of orbitals: (f) $\psi_{1 \downarrow}-\psi_{2 \downarrow}$, (g) $\psi_{3 \uparrow}-\psi_{5 \uparrow}$, (h) $\psi_{6 \uparrow}-\psi_{8 \uparrow}$, (i) $\psi_{3 \downarrow}-\psi_{8 \downarrow}$ and (j) $\psi_{9 \uparrow}-\psi_{10 \uparrow}$. Scale bar: $5 \AA$. 